The autopsy showed compression, principally of the posterior roots, with some ascending degeneration in the cord. The peripheral nerves were intact, and an alcoholic sensory polyneuritis was thereby excluded.

2. Hepatic Function and Depression.-Cololian first considers the relationship between liver disease and melancholia, and holds that the latter, instead of being a pure psychosis, is a disease of the entire organism and presents physical symptoms and signs wrich are referable principally to affections of the liver. He then traces the parallelism between the two conditions, and notes that in each are found xanthoderma; dyspepsia, boulimia, constipation; insomnia, migraine; sexual weakness, bradycardia, urobilinuria, etc. The third section deals with hepatopathic and neuropathic heredity among melancholiacs. The family histories show jaundice, cirrhosis and gall stones. The personal histories include jaundice of the new-born, catarrhal and lithiasal jaundice.. Both histories also show clear evidence of mental degeneracy. The author believes that the degeneracy affects the liver as well as the brain, and that, with this predisposition, melancholia easily arises from the stress of an exciting cause like infectious fevers, puberty, pregnancy, etc.

3. Hospital Management.-Bourneville reports the following action of the Council Superior on his recommendations. The average number of patients under one physician should not exceed 400 , if the annual admissions do not exceed 200 . For every 100 admissions one interne should be allowed. The chief physician should have charge of the admissions and curable cases; the assistant physicians, the chronic diseases. Internes should be enlisted by concours, and should be paid at least $\$ 200$ a year, with an increase of $\$ 25$ yearly. The salary of assistant physicians should be increased $\$ 125$ a year. Complete hospital reports should be published each year. H. J. NichoLs (Washington).

\title{
Centralblatt für Nervenheilkunde und Psychiatrie
}

(Vol. 28, 1905, No. 187, April 15.)

\section{An Hypothesis Concerning the Nature of the Katatonic Symptom- Complex. LundBorg.}

2. Unilateral Hippus in Progressive Paresis. GaUpP.

I. Katatonic Symptom-Complex.-Lundborg discusses the relation between the so-called motor-neuroses and a condition of insufficiency of tne parathyroid glands. The theory is illustrated diagramatically by a chain in which, on one side, is indicated the association of thyroid insutficiency with myxedema and a condition of stupor; of thyroid hyperfunction with Basedow's disease and psychic conditions. On the other side of the chain, insufficiency of the parathyroids as related to the katatonic symptom-complexes-paralysis agitans, tetany, myoclonus-epilepsy and myotonia; hyper-function of the parathyroids-with myasthenic paralysis and family periodic paralysis. This theory was discussed in a previous paper. The results of experiments by Blum are quoted at length, relating the production of psychic disturbances in dogs after removal of the thyroid. Also an experiment by Berger in which a dog was injected with serum from a katatonic patient, producing tremor, clonic muscle contractions, and finally stupor with katatonia. Lundborg believes that there exists a distinct endogenous toxin which caused the symptoms described also, that the myoclonic contractions and the katatonic symptoms are related to the toxin.

Based upon this, he believes the katatonic symptom-complex of tetany and myoclonus to depend upon a distinct insufficience of the parathyroid glands. Also, that the psychic symptoms present in dementia præcox depend, perhaps, upon an altered chemistry of the thyroid gland "Insuffizierung und Dysfunction"). The above-mentioned diagram is, therefore, enlarged, including within the varieties of katatonia, myxedema, 
Basedow's disease, paralysis agitans and paralytic myasthenia.

2. Unilateral Hippus. - Gaupp notes the lack of unanimity concerning the meaning of the term "hippus." He uses the term to indicate a slow, rhythmical contraction and dilatation of the pupil, occurring without the stimulus of any of the known causes of pupillary movements. (Light, darkness, convergence, accommodation, pain.) After citing instances in which hippus has been observed by others, Gaupp relates a case in which, following a blow on the head, there developed typical symptoms of advanced progressive paralysis. The left pupil was insensible to all forms of stimuli, but showed a rhythmical variation in its diameter, I-7 contractions occurring in 15 seconds.

\section{(Vol. 28, 1905, No. 188, May r.)}

I. On the Importance of Attention to the Localization and Development of Hallucinatory Pictures. V. BECHTEREw.

2. Contribution to the Symptomatology of Chronic Progressive Chorea.

I. Hallucinatory Pictures.-The calling up and localization of hallucinatory pictures by attracting the patient's attention to an actual stimulation like that which comprises the hallucination, according to Bechterew, is made possible by experiment; also was he able to change the location of hallucinatory sounds in alcoholics with hallucinations. This was accomplished by attracting the patient's attention to the sound produced by the vibration of an induction apparatus, when the same sounds which he has heard elsewhere seemed to him to come from the induction apparatus.. Wherever the attention of the patient be drawn, there will he hear the hallucinatory sound. The same state of affairs prevails when, in an hysteric, auditory hallucinations are suggested during hypnotic sleep. Similar phenomena occur in visual hallucinations. When a single variety of hallucination existed, e. g., auditory, it was, as a rule, impossible to suggest hallucinations in other organs. The involuntary attraction of the attention, therefore, can be made a means of the production of hallucinations.

2. Chronic Progressive Chorea.-Liebers reports a case, with typical symptoms of Huntington's chorea, giving an entirely negative history concerning any form of neurotic tendency in her antecedents, but in whose daughter appear feeble-mindedness and muscular twitching in fingers and face. The case described presented marked speech disturbance and dementia with occasional passionate outbursts. Dermographia and a diffuse erythema appeared during the periods of excitement, together with a slight right-sided paresis.

$$
\text { (Vol. 28, 1905, No. 189, May 17.) }
$$

I. Tabes and Synergy. Catola and Lewandowsky.

2. On a Specific Antitoxine in the Blood Serum of Epileptics. CENI.

I. Tabes and Synergy.-Based upon the observation of cases with marked ataxia, the writers found that patients in which the ataxia was present in the upper extremities retained the muscle synergy in the flexors and extensors; that is, in clenching the fists and opening the closed fist. The writers' observations disagree with those of Foerster that muscle synergy depends upon the integrity of sensibility.

2. Blood Serum of Epileptics.-In a previous contribution Ceni demonstrated that the epileptogenous serum possessed a definite specificity for man, but not for other species of animals; also, that it varied with the intensity of the disease; $e$. g., the status epilepticus. In the present investigation, the results of which he publishes in this paper, experiments were made with the "natural antitoxic substances of the sera of epileptics." He shows that the antitoxic substances of the blood of epileptics have the property of neutralizing the toxic action of the epileptogenous sera of man. Three series of experiments were made: 
I. With serum of epileptics durin the various phases of the regular course of the disease; $i$. $e$., before after and during the attack, and during the interval. Serum from a patient having attacks every $20-25$ days was mixed with antiserum $(0.5 \mathrm{ccm}$. in $10 \mathrm{ccm}$. of the former) and injected into another epileptic. The effect was the same in every case, namely, that the $\mathrm{ccm}$. of serum neutralized the toxic effect of the $0.5 \mathrm{ccm}$. of the antiserum, in greater part or entirely.

2. With serum of severe cases (status epilepticus). This serum was hypotoxic; injected alone, it never produced signs of acute poisoning; on the contrary, almost without toxic effect. When $10 \mathrm{ccm}$. of the serum from this patient, with $1 / 2 \mathrm{ccm}$., also $1 / 4 \mathrm{ccm}$. antiserum, injected into another epileptic, produced at once the symptoms of an acute specific poisoning.

3. Experiments with the serum of epileptics, which following the injection of specific antiserum, also following the injection of hypertoxic serum of other epileptics, showed an aggravation of the disease, and at the same time a diminution of the antitoxic power of the blood-serum of these patients against the specific antiserum.
A. C. Buckley (Philadelphia).

\section{Miscellany}

The Non-Toxic Amblyopras. T. W. Moore, Huntington, W. Va. (Journal A. M. A., Aug. 26).

The author classes the non-toxic amblyopias under three heads: 1 . Those cases of amblyopia exanopsia held to be due to inabjlity to use corresponding areas of the retina at the same time. This may be due to high refractive errors, to improper muscle balance or to non-development of the cerebral fusion center. 2. The hysterical amblyopias. 3. The somewhat similar but different group characterized by impaired vision, contracted fields for white and normal color fields, the color vision being proportionate to the general visual acuity; these cases are usually designated as anesthesia of the retina. Lastly, there is a sub-group of these latter due to traumatism, often with pathologic changes of the fundus from old hemorrhage or choroidal rupture that has left the parts uninvolved in a healthy condition, though vision has not returned. These cases are closely allied to the preceding group, differing only in etiology and in the limited results of treatrinent. Amblyopia exanopsia is usually easy of diagnosis, the defective vision, apparently normal fundus, frequent squint of one eye and the high refractive error making it clear from the first. Moore finds the convex-glass cure of Fronmuller the most satisfactory treatment, and reproduces the directions in full. In some of his cases he has had surprisingly good results, and some improvement in all in which he could command the coopperation of the patient. With the hysterical amblyopias the diagnosis is also, as a rule, not difficult. The concentric and constant narrowing of the visual field with reversal for colors is characteristic. Other conditions may show the contraction, but not with the color reversal. In addition, there are the other signs of hysteria, both ocular and otherwise, to aid in the diagnosis. The treatment consists in general measures to build up the patient's health, correction of the hysterical tendency and of any errors of refraction, etc. As regards the cases grouped by him in his third class, and which fall etiologically under two heads, those due to traumatism and those of unknown causation, Moore disagrees with Charcot and Leber as to these being hysterical, and holds that the trouble is really in the retina instead of in the brain. An illustrative case is reported. His treatment in these cases is electricity, and he finds the faradic and high-frequency currents equally as efficient as the galvanic, and the high-frequency current perhaps the most useful. He applies the electrode over the closed lids, usually using the unipolar, 